\title{
High-Energy Zero-Norm States and Symmetries of String Theory
}

\author{
Chuan-Tsung Chan, ${ }^{1, *}$ Pei-Ming Ho, ${ }^{2, \dagger}$ Jen-Chi Lee, ${ }^{3, \ddagger}$ Shunsuke Teraguchi, ${ }^{4, \S}$ and Yi Yang ${ }^{3, \|}$ \\ ${ }^{1}$ Physics Division, National Center for Theoretical Sciences, Hsinchu, Taiwan, Republic of China \\ ${ }^{2}$ Department of Physics, National Taiwan University, Taipei, Taiwan, Republic of China \\ ${ }^{3}$ Department of Electrophysics, National Chiao-Tung University, Hsinchu, Taiwan, Republic of China \\ ${ }^{4}$ Physics Division, National Center for Theoretical Sciences, Taipei, Taiwan, Republic of China
}

(Received 28 May 2005; published 2 May 2006)

\begin{abstract}
High-energy limit of zero-norm states in the old covariant first quantized spectrum of the 26D open bosonic string, together with the assumption of a smooth behavior of string theory in this limit, are used to derive infinitely many linear relations among the leading high-energy, fixed-angle behavior of four-point functions of different string states. As a result, ratios among all high-energy scattering amplitudes of four arbitrary string states can be calculated algebraically and the leading order amplitudes can be expressed in terms of that of four tachyons as conjectured by Gross in 1988. A dual calculation can also be performed and equivalent results are obtained by taking the high-energy limit of Virasoro constraints. Finally, we compute all high-energy scattering amplitudes of three tachyons and one massive state at the leading order by saddle-point approximation to verify our results.
\end{abstract}

PACS numbers: $11.25 . \mathrm{Db}, 11.25 . \mathrm{Hf}$

One of the fundamental issues in string theory is its space-time symmetry. In local quantum field theories, a symmetry principle is usually postulated, which can be used to fix the interactions in the theory. In string theory, on the contrary, it is the interaction, prescribed by the very tight quantum consistency conditions due to the extendedness of strings, which determines the symmetry. For example, the massless gauge symmetries of 10D Heterotic string [1] were discovered to be $S O(32)$ or $E_{8}^{2}$ by the string one-loop consistency or modular invariance of the theory. Symmetries with parameters containing both Yang-Mills and Einstein indices were found explicitly at massive levels [2]. Being a consistent quantum theory with no free parameter and an infinite number of states, it is conceivable that there exists a huge symmetry group, which is responsible for the ultraviolet finiteness of string theory.

Historically, a key progress to understand the symmetry of string theory is to study the high-energy, fixed-angle behavior of string scattering amplitudes [3-5]. This is strongly motivated by the success on the study of the high-energy behavior of a local quantum field theory, for example, the renormalization group and the discovery of asymptotic freedom in QCD [6]. Moreover, the spontaneously broken symmetries are often hidden at low energy, but become evident at high energies. Other approaches related to this development include the studying the highenergy, fixed momentum transfer regime [7], vertex operator algebra for compactified space time or on a lattice [8], or the Hagedorn transition at high temperature [9], and taking the tensionless limit of the worldsheet theory [10]. Despite all these efforts, a concrete picture of the underlying symmetry of strings has not emerged.

Recently, an algebraic approach $[11,12]$ was developed to derive linear relations among correlation functions in the high-energy limit, which are presumably a manifestation of the hidden symmetry. An important ingredient of this approach is the zero-norm states in the old covariant first quantization (OCFQ). In fact, it can be shown that [13] offshell gauge transformations of Witten string field theory, after imposing the no-ghost condition, are identical to the on-shell stringy gauge symmetries generated by two types of zero-norm states in the generalized massive $\sigma$ model approach of string theory [14]. The corresponding on-shell Ward identities were also constructed in Ref. [15]. The connection between zero-norm states and the space-time symmetry was further elucidated in the case of $2 \mathrm{D}$ string where the discrete zero-norm states were shown [16] to generate a $\omega_{\infty}$ symmetry algebra [17]. Furthermore, these discrete zero-norm states approach the discrete Polyakov positive-norm states in the high-energy limit [18].

At first sight one may raise the objection that, while the zero-norm states correspond to gauge transformations, they cannot relate physically inequivalent states. The trick is to deform zero-norm states to some positive-norm states, which will nevertheless be called high-energy zero-norm states (HZNS's). It is the decoupling of the HZNS in the high-energy limit that will allow us to derive nontrivial relations among inequivalent amplitudes. It was emphasized in Refs. $[18,19]$ that the decoupling of the HZNS in the high-energy limit is a nontrivial assumption. Those who are interested in a more detailed account of the assumption please see Refs. [18,20]. Roughly speaking, this assumption is about the regularity of the high-energy limit of string theory.

In this Letter, we will generalize the calculations for the first few mass levels $[11,12,19]$ to arbitrary mass levels for the open bosonic string. Infinite linear relations among high-energy scattering amplitudes of different string states at fixed but arbitrary mass levels will be obtained. These linear relations are then used to determine uniquely the proportional constants among high-energy scattering amplitudes of different string states to the leading order. Based 
on the high-energy scattering amplitudes for certain representative states obtained in Refs. [11,19], one can then derive the general formula of high-energy scattering amplitude for four arbitrary string states, and express them in terms of that of four tachyons. It was first conjectured by Gross in 1988 [4] that scattering amplitudes of arbitrary states are linearly related to each other in the high-energy limit. These linear relations hint at the existence of an infinite space-time string symmetry hidden at low energy $[4,21]$ but gets restored at high energies.

Let us now explain the first approach in detail. In the OCFQ spectrum of open bosonic string theory, the solutions of physical state conditions include positive-norm propagating states and two types of zero-norm states. The latter are (we use the notation in Refs. [22])

Type I : $L_{-1}|x\rangle, \quad$ where $L_{1}|x\rangle=L_{2}|x\rangle=0, \quad L_{0}|x\rangle=0$;

Type II: $\left(L_{-2}+\frac{3}{2} L_{-1}^{2}\right)|\tilde{x}\rangle, \quad$ where $L_{1}|\tilde{x}\rangle=L_{2}|\tilde{x}\rangle=0$,

$$
\left(L_{0}+1\right)|\tilde{x}\rangle=0 \text {. }
$$

While type I states have zero norm for any space-time dimensions, type II states have zero norm only for $D=$ 26. We take the second massive level $M^{2}=4$ as an example to illustrate our approach. The first step is to list the stringy Ward identities for four-point functions derived from the decoupling of all zero-norm states at this mass level [15]

$$
\begin{gathered}
k_{\mu} \theta_{\nu \lambda} \mathcal{T}_{\chi}^{(\mu \nu \lambda)}+2 \theta_{\mu \nu} \mathcal{T}_{\chi}^{(\mu \nu)}=0 \\
\left(\frac{5}{2} k_{\mu} k_{\nu} \theta_{\lambda}^{\prime}+\eta_{\mu \nu} \theta_{\lambda}^{\prime}\right) \mathcal{T}_{\chi}^{(\mu \nu \lambda)} \\
+9 k_{\mu} \theta_{\nu}^{\prime} \mathcal{T}_{\chi}^{(\mu \nu)}+6 \theta_{\mu}^{\prime} \mathcal{T}_{\chi}^{\mu}=0, \\
\left(\frac{1}{2} k_{\mu} k_{\nu} \theta_{\lambda}+2 \eta_{\mu \nu} \theta_{\lambda}\right) \mathcal{T}_{\chi}^{(\mu \nu \lambda)} \\
+9 k_{\mu} \theta_{\nu} \mathcal{T}_{\chi}^{[\mu \nu]}-6 \theta_{\mu} \mathcal{T}_{\chi}^{\mu}=0, \\
\left(\frac{17}{4} k_{\mu} k_{\nu} k_{\lambda}+\frac{9}{2} \eta_{\mu \nu} k_{\lambda}\right) \mathcal{T}_{\chi}^{(\mu \nu \lambda)} \\
+\left(21 k_{\mu} k_{\nu}+9 \eta_{\mu \nu}\right) \mathcal{T}_{\chi}^{(\mu \nu)}+25 k_{\mu} \mathcal{T}_{\chi}^{\mu}=0,
\end{gathered}
$$

where $\theta_{\mu \nu}$ is an arbitrary symmetric, transverse, and traceless polarization tensor, and $\theta_{\lambda}^{\prime}$ and $\theta_{\lambda}$ are arbitrary transverse vectors. $\mathcal{T}^{\prime}{ }^{\prime} s$ in Eqs. (3)-(6) are $\chi$ th order stringloop four particle scattering amplitudes with, say, the second vertex $V_{2}\left(k_{2}\right)$ constructed from zero-norm states at the mass level $M^{2}=4$ and $k_{\mu} \equiv k_{2 \mu}$. For example, Eq. (3) states the decoupling of the zero-norm state corresponding to the vertex operator $V_{2}(k)=\left(k_{\mu} \theta_{\nu \lambda} \partial X^{\mu} \partial X^{\nu} \partial X^{\lambda}+\right.$ $\left.2 \theta_{\mu \nu} \partial^{2} X^{\mu} \partial X^{\nu}\right) e^{i k \cdot X}$, and

$$
\begin{aligned}
\mathcal{T}_{X}^{(\mu \nu \lambda)} & =\left\langle V_{1}\left(\partial X^{\mu} \partial X^{\nu} \partial X^{\lambda} e^{i k \cdot X}\right) V_{3} V_{4}\right\rangle_{\chi}, \\
\mathcal{T}_{\chi}^{(\mu \nu)} & =\left\langle V_{1}\left(\partial^{2} X^{(\mu} \partial X^{\nu)} e^{i k \cdot X}\right) V_{3} V_{4}\right\rangle_{\chi} .
\end{aligned}
$$

The rest of the vertices $V_{1}, V_{3}$, and $V_{4}$ in $\mathcal{T}_{X}^{\prime} s$ can be arbitrary string states and their tensor indices are omitted.
Note that Eqs. (3)-(6) are valid to all loops $\chi$ and at all energies $E$. We use labels 1 and 2 for incoming particles and 3 and 4 for outgoing particles. In the center of mass frame, the scattering angle $\phi_{\text {c.m. }}$ is defined to be the angle between $\vec{k}_{1}$ and $\vec{k}_{3}$.

To enumerate all possible polarizations $\theta_{\mu \nu}, \theta_{\mu}, \theta_{\mu}^{\prime}$ for these equations, we define a basis of polarization vectors for the second vertex. We have $e^{P}=\frac{1}{M_{2}}\left(E_{2}, k_{2}, 0, \ldots\right)=\frac{k_{2}}{M_{2}}$ as the momentum polarization, $e^{L}=\frac{1^{2}}{M_{2}}\left(k_{2}, E_{2}, 0, \ldots\right)$ the longitudinal polarization, and $e^{T}=(0,0,1, \ldots)$ the transverse polarization. Note that $e^{P}$ approaches to $e^{L}$ in the high-energy limit, and the scattering plane is defined by the spatial components of $e^{L}$ and $e^{T}$. Polarizations perpendicular to the scattering plane are ignored because they are kinematically suppressed for four-point scatterings in the high-energy limit.

The next step is to consider all possible polarizations for these equations and to replace $e^{P}$ by $e^{L}$ in the high-energy limit. To the leading order, Eqs. (3)-(6) imply (we drop loop order $\chi$ here to simplify the notation) $[11,12]$

$$
\begin{gathered}
\mathcal{T}_{L L T}+\mathcal{T}_{(L T)} \simeq 0, \\
10 \mathcal{T}_{L L T}+\mathcal{T}_{T T T}+18 \mathcal{T}_{(L T)} \simeq 0, \\
\mathcal{T}_{L L T}+\mathcal{T}_{T T T}+9 \mathcal{T}_{[L T]} \simeq 0,
\end{gathered}
$$

where the subscripts denote the polarizations. These linear equations can be easily solved,

$$
\mathcal{T}_{T T T}: \mathcal{T}_{L L T}: \mathcal{T}_{(L T)}: \mathcal{T}_{[L T]} \simeq 8: 1:-1:-1
$$

After some simple power counting of the energy order for all amplitudes [11], one concludes that all other amplitudes with any $V_{2}$ at the same mass level (but with $V_{1,3,4}$ fixed) are subleading, as compared with the amplitudes already appearing in the linear relation above. That is, the decoupling of the HZNS gives complete information about all amplitudes at the leading order in the high-energy limit.

This linear relation among scattering amplitudes agrees with direct computation. For example, consider the fourpoint functions with one tensor $V_{2}$ and three tachyons $V_{1,3,4}$. The $s-t$ channel contributions to the scattering amplitudes in Eq. (11) are given by [11,12] $\mathcal{T}_{T T T} \simeq$ $-8 E^{9} \sin ^{3} \phi_{C M} \mathcal{T}(3) \simeq 8 \mathcal{T}_{L L T} \simeq-8 \mathcal{T}_{(L T)} \simeq-8 \mathcal{T}_{[L T]}$, where $\mathcal{T}(3)$ is given by

$$
\begin{aligned}
\mathcal{T}(n)= & \sqrt{\pi}(-1)^{n-1} 2^{-n} E^{-1-2 n}\left(\sin \frac{\phi_{\text {c.m. }}}{2}\right)^{-3} \\
& \times\left(\cos \frac{\phi_{\text {c.m. }}}{2}\right)^{5-2 n} \\
& \times \exp \left(-\frac{s \ln s+t \ln t-(s+t) \ln (s+t)}{2}\right),
\end{aligned}
$$

which is the high-energy limit of $\left[\Gamma\left(-\frac{s}{2}-1\right) \Gamma\left(-\frac{t}{2}-1\right)\right] /$ $\Gamma\left(\frac{u}{2}+2\right)$ with $s+t+u=2 n-8$. Here $s=-\left(k_{1}+k_{2}\right)^{2}$, $t=-\left(k_{2}+k_{3}\right)^{2}$ and $u=-\left(k_{1}+k_{3}\right)^{2}$ are the Mandelstam variables [12]. 
The aim of this Letter is to show that there is indeed only one independent component of high-energy scattering amplitude at the leading order for general mass levels, and to calculate the proportional constants generalizing Eq. (11). One first notes that the decoupling of HZNS indicates that the only states that will survive the highenergy limit at level $M^{2}=2(n-1)$ are of the form

$$
|n, 2 m, q\rangle \equiv\left(\alpha_{-1}^{T}\right)^{n-2 m-2 q}\left(\alpha_{-1}^{L}\right)^{2 m}\left(\alpha_{-2}^{L}\right)^{q}|0, k\rangle .
$$

It is algebraically proved in Refs. $[18,20]$ that an amplitude becomes subleading if we replace a state of this form by another state not of this form at the same mass level. One can also verify this fact by explicitly calculating an amplitude using saddle-point method $[18,19]$.

The next step is to use the decoupling of two types of HZNS

$$
\begin{aligned}
L_{-1}|n-1,2 m-1, q\rangle \simeq & M|n, 2 m, q\rangle+(2 m-1) \\
& \times|n, 2 m-2, q+1\rangle, \\
L_{-2}|n-2,0, q\rangle \simeq & \frac{1}{2}|n, 0, q\rangle+M|n, 0, q+1\rangle,
\end{aligned}
$$

to deduce the ratios of all amplitudes at the leading order. The final result is [18]

$$
\mathcal{T}^{(n, 2 m, q)}=\left(-\frac{1}{M}\right)^{2 m+q}\left(\frac{1}{2}\right)^{m+q}(2 m-1) ! ! \mathcal{T}^{(n, 0,0)} .
$$

Equation (16) correctly reproduces the proportional constants for mass level $M^{2}=4,6$ after the Young tableaux decomposition $[11,12]$.

Equation (16) also allows us to obtain the general formula for four particle scattering amplitudes at the tree level in the high-energy limit

$$
\begin{aligned}
\left\langle V_{1} V_{2} V_{3} V_{4}\right\rangle= & \prod_{i=1}^{4}\left(-\frac{1}{M_{i}}\right)^{2 m_{i}+q_{i}}\left(\frac{1}{2}\right)^{m_{i}+q_{i}} \\
& \times\left(2 m_{i}-1\right) ! ! \mathcal{T}_{n_{1} n_{2} n_{3} n_{4}}^{T^{1} \cdot T^{2} \cdot T^{3} \cdot T^{4} \cdot .}
\end{aligned}
$$

where $\mathcal{T}_{n_{1} n_{2} n_{3} n_{4}}^{T^{1} \cdot T^{2} \cdot T^{4} \cdot .}$ is the high-energy scattering amplitude for $V_{i}=\left(e^{T^{i}} \cdot \partial X\right)^{n_{i}} e^{i k_{i} X_{i}}(i=1, \ldots, 4)$. ( $T^{i}$ is the transverse polarization for the $i$ th particle. $e^{T}$ defined earlier is denoted $e^{T^{2}}$ here.) It only depends on the sum $n=$ $\sum_{i=1}^{4} n_{i}$ and is given by $[11,19]$

$$
\begin{aligned}
\mathcal{T}_{n_{1} n_{2} n_{3} n_{4}}^{T^{1} \cdot T^{2} \cdot T^{3} \cdot T^{4} \cdot .} & =\left[-2 E^{3} \sin \phi_{C M}\right]^{\sum n_{i}} \mathcal{T}\left(\sum n_{i}\right) \\
& =2 \sqrt{\pi} e^{n-4}(s t u)^{(n-3) / 2} e^{-(1 / 2)(s \ln s+t \ln t+u \ln u)} .
\end{aligned}
$$

It is interesting to see that the contribution of the second term $\frac{3}{2} L_{-1}^{2}|\tilde{x}\rangle$ of type II zero-norm states in Eq. (2) to the stringy Ward identities is negligible in the high-energy limit. This hints at a "dual" calculation, the Virasoro constraints, to derive Eq. (16) [18]. It is convenient to use the Young tableaux to handle the complicated symmetric structures of tensor fields with higher spins. The most general state in the mass level $M^{2}=2(n-1)$ can be written as

$$
|n\rangle=\left\{\sum_{m_{j}} \stackrel{k}{\otimes} \frac{1}{j=1} \frac{\mu^{m_{j} m_{j}} !}{\mu_{1}^{j}|\cdots| \mu_{m_{j}}^{j}} \alpha_{-j}^{\mu_{1}^{j}} \cdots \alpha_{-j}^{\mu_{m_{j}}^{j}}\right\}|0, k\rangle,
$$

where $m_{j}$ is the number of the operator $\alpha_{-j}^{\mu}$ and the summation runs over all possible combinations of $m_{j}$ with $\sum_{j=1}^{k} j m_{j}=n$. Since the upper indices $\left\{\mu_{1}^{j} \ldots \mu_{m_{j}}^{j}\right\}$ for $\alpha_{-j}^{\mu_{1}^{j}} \ldots \alpha_{-j}^{\mu_{m_{j}}^{j}}$ are symmetric, we can use the 1-row Young tableaux to stand for the coefficients in (19). The direct product $\otimes$ acts on the Young tableaux in the standard way. We have also normalized the state by factors $1 /\left(j^{m_{j}} m_{j} !\right)$. Next, we will apply the Virasoro constraints to the state (19). The only Virasoro constraints which need to be considered are $L_{1}|n\rangle=L_{2}|n\rangle=0$. They give, in the high-energy limit [18],

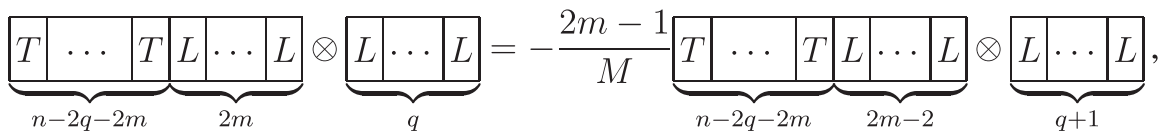

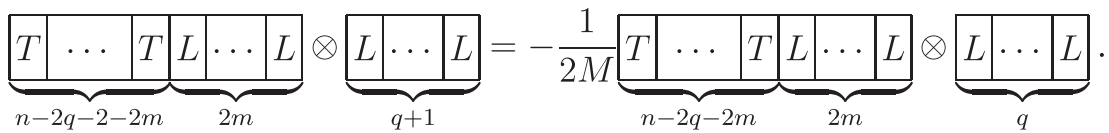

It is worth emphasizing that these equations are equivalent to Eqs. (14) and (15) in the first approach. Combining Eqs. (20a) and (20b) gives our main result Eq. (16).

Finally, we compute all high-energy scattering amplitudes of three tachyons and one massive state Eq. (13) by saddlepoint approximation [19] to justify our result Eq. (16). The $s-t$ channel contribution to the high-energy scattering amplitude at tree level is

$$
\mathcal{T}^{(n, 2 m, q)} \simeq \int_{0}^{1} d x x^{k_{1} \cdot k_{2}}(1-x)^{k_{2} \cdot k_{3}}\left[\frac{e^{T} \cdot k_{1}}{x}-\frac{e^{T} \cdot k_{3}}{1-x}\right]^{n-2 m-2 q} \cdot\left[\frac{e^{P} \cdot k_{1}}{x}-\frac{e^{P} \cdot k_{3}}{1-x}\right]^{2 m}\left[-\frac{e^{P} \cdot k_{1}}{x^{2}}-\frac{e^{P} \cdot k_{3}}{(1-x)^{2}}\right]^{q},
$$

where we have substituted the polarization $L$ by $P$. In order to apply the saddle-point method, we rewrite the amplitude 
above in the form $\mathcal{T}^{(n, 2 m, q)}(K)=\int_{0}^{1} d x u(x) e^{-K f(x)}$, where $K \equiv-k_{1} \cdot k_{2} \rightarrow 2 E^{2}, f(x) \equiv \ln x-\tau \ln (1-x), \tau \equiv-\left(k_{2}\right.$. $\left.k_{3}\right) /\left(k_{1} \cdot k_{2}\right) \rightarrow \sin ^{2} \frac{\phi_{\text {c.m }}}{2}$ and $u(x) \equiv\left[k_{1} \cdot k_{2} / M\right]^{q+2 m}(1-$ $x)^{-n+2 m+2 q}\left(f^{\prime}\right)^{2 m}\left(f^{\prime \prime}\right)^{q}\left(-e^{T} \cdot k_{3}\right)^{n-2 m-2 q}$. The saddle point for the integration of moduli, $x=x_{0}=\frac{1}{1-\tau}$, is defined by $f^{\prime}\left(x_{0}\right)=0$. From the definition of $u(x)$, it is easy to see that $u\left(x_{0}\right)=u^{\prime}\left(x_{0}\right)=\cdots=u^{(2 m-1)}\left(x_{0}\right)=0$ [23], and one can easily compute $u^{(2 m)}\left(x_{0}\right)$ and evaluate the Gaussian integral

$$
\begin{aligned}
\mathcal{T}^{(n, 2 m, q)}= & \int_{0}^{1} d x u(x) e^{-K f(x)} \\
= & \sqrt{\frac{2 \pi}{K f_{0}^{\prime \prime}}} e^{-K f_{0}}\left[\frac{u_{0}^{(2 m)}}{2^{k} m !\left(f_{0}^{\prime \prime}\right)^{m} K^{m}}+O\left(\frac{1}{K^{m+1}}\right)\right] \\
= & \sqrt{\frac{2 \pi}{K f_{0}^{\prime \prime}}} e^{-K f_{0}}\left[(-1)^{n-m} \frac{2^{n-q-2 m}(2 m) !}{k ! M^{q+2 m}}\right. \\
& \left.\times \tau^{-n / 2}(1-\tau)^{3 n / 2} E^{n}+O\left(E^{n-2}\right)\right] .
\end{aligned}
$$

This result shows that the high-energy four-point amplitudes of states at fixed mass level $n$ share the same energy and angular dependence, and one can compute the ratios among high-energy amplitudes to correctly reproduce Eq. (16). More details can be found in Ref. [18].

Although our discussions focus on the 26D open bosonic string theory, the same approach can be applied to superstrings as well [24]. The infinitely many linear relations which uniquely determine the ratios of all high-energy four-point functions at a fixed mass level strongly suggest the hidden symmetry of the string theory. Even though we have not identified the symmetry group for the string theory, this work sheds new light towards the final answer.

We thank Jiunn-Wei Chen, Tohru Eguchi, Koji Hashimoto, Hiroyuki Hata, Takeo Inami, Hsien-Chung Kao, Yeong-Chuan Kao, Yoichi Kazama, Yutaka Matsuo, and Tamiaki Yoneya for discussions. This work is supported in part by the National Science Council, Taiwan, R.O.C and National Center for Theoretical Sciences, Hsinchu, Taiwan, R.O.C (Grant No. NSC 94-2119-M002-001).

*Electronic address: ctchan@phys.cts.nthu.edu.tw;

†Electronic address: pmho@ntu.edu.tw;

ثElectronic address: jcclee@cc.nctu.edu.tw;

${ }^{\S}$ Electronic address: teraguch@phys.ntu.edu.tw;

"Electronic address: yiyang@mail.nctu.edu.tw

[1] D. J. Gross, J. A. Harvey, E. J. Martinec, and R. Rohm, Nucl. Phys. B256, 253 (1985); Nucl. Phys. B267, 75 (1986).

[2] J. C. Lee, Phys. Lett. B 337, 69 (1994).
[3] D. J. Gross and P. F. Mende, Phys. Lett. B 197, 129 (1987); Nucl. Phys. B303, 407 (1988).

[4] D. J. Gross, Phys. Rev. Lett. 60, 1229 (1988); Philos. Trans. R. Soc. London, Ser. A 329, 401 (1989).

[5] D. J. Gross and J. L. Manes, Nucl. Phys. B326, 73 (1989); see section 6 for details.

[6] D. J. Gross and F. Wilczek, Phys. Rev. Lett. 30, 1343 (1973); Phys. Rev. D 8, 3633 (1973); Phys. Rev. D 9, 980 (1974); H. D. Politzer, Phys. Rev. Lett. 30, 1346 (1973).

[7] D. Amati, M. Ciafaloni, and G. Veneziano, Phys. Lett. B 216, 41 (1989).

[8] G. W. Moore, hep-th/9305139; G. W. Moore, hep-th/ 9310026; P. C. West, Mod. Phys. Lett. A 10, 761 (1995); N. Moeller and P. West, Nucl. Phys. B729, 1 (2005).

[9] J. J. Atick and E. Witten, Nucl. Phys. B310, 291 (1988); R. Hagedorn, Nuovo Cimento Suppl. 3, 147 (1965).

[10] See, for instance, J. Isberg, U. Lindstrom, B. Sundborg, and G. Theodoridis, Nucl. Phys. B411, 122 (1994); B. Sundborg, Nucl. Phys. B, Proc. Suppl. 102, 113 (2001); E. Sezgin and P. Sundell, Nucl. Phys. B644, 303 (2002); B660, 403(E) (2003); C. S. Chu, P. M. Ho, and F. L. Lin, J. High Energy Phys. 09 (2002) 003; G. Bonelli, Nucl. Phys. B669, 159 (2003).

[11] C. T. Chan and J. C. Lee, Phys. Lett. B 611, 193 (2005); J. C. Lee, hep-th/0303012.

[12] C. T. Chan and J. C. Lee, Nucl. Phys. B690, 3 (2004).

[13] H. C. Kao and J. C. Lee, Phys. Rev. D 67, 086003 (2003); C. T. Chan, J.C. Lee, and Y. Yang, Phys. Rev. D 71, 086005 (2005).

[14] J. C. Lee, Phys. Lett. B 241, 336 (1990); Phys. Rev. Lett. 64, 1636 (1990); J. C. Lee and B. Ovrut, Nucl. Phys. B336, 222 (1990).

[15] J. C. Lee, Prog. Theor. Phys. 91, 353 (1994).

[16] T. D. Chung and J. C. Lee, Phys. Lett. B 350, 22 (1995); Z. Phys. C 75, 555 (1997); J. C. Lee, Eur. Phys. J. C 1, 739 (1998).

[17] J. Avan and A. Jevicki, Phys. Lett. B 266, 35 (1991); Phys. Lett. B 272, 17 (1991); I. R. Klebanov and A. M. Polyakov, Mod. Phys. Lett. A 6, 3273 (1991). E. Witten, Nucl. Phys. B373, 187 (1992); E. Witten and B. Zwiebach, Nucl. Phys. B377, 55 (1992).

[18] C. T. Chan, P. M. Ho, J. C. Lee, S. Teraguchi, and Y. Yang, Nucl. Phys. B725, 352 (2005).

[19] C. T. Chan, P. M. Ho, and J. C. Lee, Nucl. Phys. B708, 99 (2005).

[20] C. T. Chan, P. M. Ho, J. C. Lee, S. Teraguchi, and Y. Yang, hep-th/0509009.

[21] J. C. Lee, Phys. Lett. B 326, 79 (1994); M. Evans and B. A. Ovrut, Phys. Lett. B 231, 80 (1989).

[22] M. B. Green, J.H. Schwarz, and E. Witten, Superstring Theory (Cambridge University Press, Cambridge, England, 1987), Vol. 1.

[23] Notice that we need to take the $2 m$ th order saddle-point approximation in order to find the leading order coefficient of the correlation function. This explains [19] what is missing in the previous calculations [3-5].

[24] C. T. Chan, J. C. Lee, and Y. Yang, Nucl. Phys. B738, 93 (2006). 